\title{
Computation-Aware Intra-Mode Decision for H.264 Coding and Transcoding
}

\author{
Jhih-Shen Shen, Chih-Hung Chen, and Chia-Ming Tsai \\ Department of Computer Science \& Information Engineering \\ National Chung Cheng University \\ Chiayi 621, Taiwan \\ tsaicm@cs.ccu.edu.tw
}

\author{
Chia-Wen Lin \\ Department of Electric Engineering \\ National Tsing Hua University \\ Hsinchu 30013, Taiwan \\ cwlin@ee.nthu.edu.tw
}

\begin{abstract}
Nowadays, most video-enabled mobile terminals have been equipped with modern video codecs. Video communications, especially for encoding $\mathrm{H} .264$ format bit-stream, however, are usually very power-consuming, leading to rather limited communication period for mobile devices powered by batteries. Computation-aware video coding can effectively extend the battery life. In this paper, we propose a computation-aware intra mode decision for H.264 coding and transcoding applications. The proposed algorithm optimizes the visual quality by adaptively adjusting the number of prediction modes in mode decision under a given computation constraint. We introduce a new concept of computation buffer and formulate the computation control of mode decision as a rate-distortion optimization problem of computation buffer control. Experimental results show that our proposed algorithm can effectively control the computational complexity while maintaining good RD-performance and satisfying the given computation constraint.
\end{abstract}

\section{INTRODUCTION}

The explosive growth of compressed video streams and repositories which are accessible worldwide and the recent addition of new video-related standards, such as H.264/AVC [1] and MPEG-21, have stimulated research for new technologies and applications in the area of multimedia architectures, processing, and networking. Users may employ heterogeneous video-enabled terminals such as computers, TVs, mobile phones and personal digital assistants with a wide range of computational and display capabilities, energy resources, features, accessibilities, and user preferences. In recently years, mobile devices have been widely deployed. These mobile devices usually have rather limited battery power lifetime. Nowadays, most video-enabled mobile terminals have been equipped with modern video codecs. Video communications, especially for encoding H.264 format bit-stream, however, are usually very power-consuming, leading to rather limited communication period for mobile devices powered by batteries. How to use the limited power more efficiently for optimal video encoding thus becomes an important issue.

The issues about power consumption reduction and effective power allocation for handheld devices have been addressed in the literature. In [2] and [3], Kannangara et al. proposed a variable complexity algorithm for H.264, and adapted for a per-frame computational control algorithm. This computational control algorithm is based on minimizing the Lagrange Rate-DistortionComplexity cost of the encoder. In [4], Wang et al. proposed a complexity adaptive motion estimation and mode decision (CAMED) method for an H.264 encoder. By giving the bit rate and computational constraints, CAMED explores the trade-off between video quality and computation resource consumption to determine the optimal motion vectors and block modes used in the motion-compensation process in the decoder.

Computation-aware video coding can effectively extend the battery life of mobile devices. In this paper, we propose a computation-aware intra mode decision for H.264 coding and transcoding applications. The proposed algorithm optimizes the visual quality by adaptively adjusting the number of prediction modes in mode decision under a given computation constraint. We introduce a new concept of computation buffer and formulate the computation control of mode decision as a rate-distortion optimization problem of computation buffer control.

\section{PROPOSED COMPUTATION CONTROL ALGORITHM}

In H.264, if all frames are intra-coded, the main complexity bottleneck is the number of Intra_ $4 \times 4$ candidate modes for RDO computation. Our algorithm is aimed at dynamically controlling the encoding complexity subject to a given computing power constraint (i.e., the remaining battery power budget) while maintaining the video quality. We define the computation constraint as the number of prediction modes to be used per $4 \times 4$ intra block. For example, a “44\%" computation constraint means that, on average four out of nine prediction modes will be chosen per $4 \times 4$ intra block in RDO computation of mode decision. With the proposed algorithm, an encoder can adaptively determine the number of candidate modes for each $4 \times 4$ intra block according to the block's characteristics under the given computation constraint. The proposed algorithm is divided into four steps as elaborated below.

\section{A. Computation Buffer Initialization}

Before encoding a video sequence, a computation buffer is set up based on a given computing power constraint. The computation buffer records and updates the available computing power budget up to the current block. Two control parameters are used:

1) $C$ : the number of remaining non-encoded $4 \times 4$ intra blocks of the video sequence.

2) Buffer: the number of avalible candidate modes that can be chosen for RDO computation. 
For instance, given "70\%" computing power constraint for a QCIF format sequence with 50 I-frames, two control parameters are determined as below:

$$
\begin{aligned}
& C=\frac{176}{4} \times \frac{144}{4} \times 50=79200, \\
& \text { Buffer }=C \times 9 \times 70 \%=498960
\end{aligned}
$$

\section{B. Modeling}

The proposed computation model is used to determine how many candidate modes can be used for predicting each $4 \times 4$ intrablock based on the features of the block. In our model, an intrablock is characterized based on the following three observations.

1) Observation 1: the rank of best mode's prediction error.

RDO computation, rather than the prediction error of prediction modes, has been used for determining the best intramode in the H.264 reference software for sake of accuracy. The RDO computation, however, consumes heavy computing power. To show the relationship between the best mode obtained by RDO and the SAD/SATD prediction errors, the SAD/SATD prediction errors of nine prediction modes are sorted by the error values. Figure 1 illustrates the percentages of the rank of the SAD and SATD prediction error (among the nine prediction modes) of the best mode obtained by mode decision with RDO for four test sequences. The figures show that the percentage of the four top-ranking prediction modes (i.e., with smallest SAD or SATD values) being chosen as the best coding mode after mode decision with RDO is about $90 \%$. Such high percentages imply that SAD or SATD prediction error can be used to predict the best mode efficiently without sacrificing the accuracy significantly. In our method, SATD is adopted to calculate the prediction error as it has better accuracy than SAD in predicting the best coding mode.

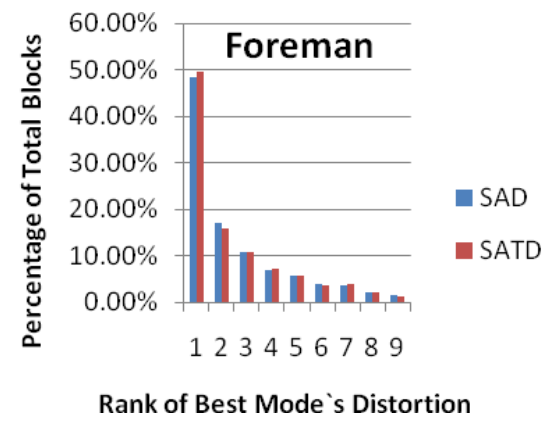

(a)

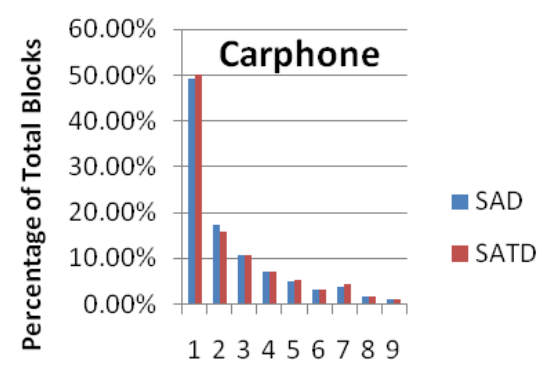

Rank of Best Mode`s Distortion

(b)

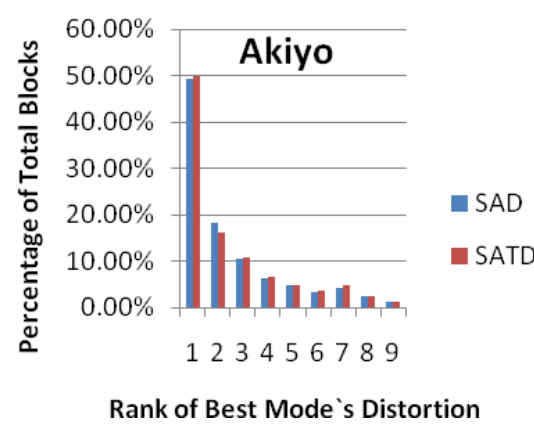

(c)

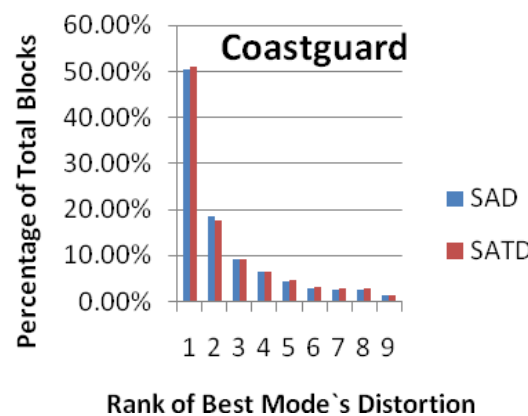

(d)

Figure 1. Average percentages of the prediction modes that are ranked by their prediction errors being chosen as the best coding mode after mode decision with RDO for four test sequences: (a) Foreman, (b) Carphone, (c) Akiyo, and (d) Coastguard.

2) Observation 2: the relationship bewteen the standard deviation of SATD and the rank.

The standard deviation $\sigma$ of nine prediction modes' SATD values can be obtained from (1):

$$
\sigma=\sqrt{\frac{1}{9} \sum_{k=0}^{8}\left(S A T D_{k}-\overline{S A T D}\right)^{2}} \text {, and } \overline{S A T D}=\frac{1}{9} \sum_{i=0}^{8} S A T D_{k}
$$

where $k$ is the index of prediction modes.

These statistics can be used to characterize the relationship between the standard deviation of SATD prediction errors and the average rank of best mode's SATD value. For example, if we find that the average rank of the best mode's SATD is " 4 " when the standard deviation of SATD prediction errors is " 50 ," the best mode can be found from the four top-ranking modes(sorted by SATD) with a high probability if the standard deviation of SATD prediction errors is " 50 ." With such models, we are able to use the statistics of SATD prediction errors of each block to estimate the average rank of best mode's SATD value without resorting to RDO computation, leading to significant computation reduction.

We use the linear regression to find the mathematical model to approximate the relationship curve as follows.

$$
\begin{cases}\text { Optimal }_{i}=a+b \cdot \sigma, & \text { for } \sigma=0 \\ \text { Optimal }_{i}=c+d \cdot \ln \sigma, & \text { for } \sigma>0\end{cases}
$$

where Optimal $_{i}$ denotes the optimal number of candidate modes. The mode parameter sets $(a, b)$ and $(c, d)$ are determined using the least squares method, as shown in (3). Table I lists the derived 
coefficient set $(a, b)$ and $(a, b)$ by using (3) in the Foreman and Carphone sequences.

$$
\begin{aligned}
& M=\left[\begin{array}{cc}
1 & \sigma_{1} \\
1 & \sigma_{2} \\
\vdots & \vdots \\
1 & \sigma_{n}
\end{array}\right] \text { and } y=\left[\begin{array}{c}
N_{1} \\
N_{2} \\
\vdots \\
N_{n}
\end{array}\right] \text { and } v=\left[\begin{array}{l}
a \\
b
\end{array}\right] \text { or }\left[\begin{array}{l}
c \\
d
\end{array}\right] \\
& \Rightarrow v=\left(M^{T} M\right)^{-1} M^{T} y
\end{aligned}
$$

TABLE I. The coefficient sets for 2 different test sequences with four different quantization parameters.

\begin{tabular}{|c|c|c|c|c|c|}
\hline Sequence & QP & $\boldsymbol{a}$ & $\boldsymbol{b}$ & $\boldsymbol{c}$ & $\boldsymbol{d}$ \\
\hline \multirow{4}{*}{ Foreman } & 28 & 2.3688 & 0.9272 & 3.6651 & -0.3942 \\
\cline { 2 - 6 } & 32 & 2.3493 & 0.9411 & 4.2650 & -0.4981 \\
\cline { 2 - 6 } & 36 & 2.1755 & 1.0469 & 5.0111 & -0.6179 \\
\cline { 2 - 6 } & 40 & 2.0805 & 1.0266 & 5.6999 & -0.7094 \\
\hline \multirow{5}{*}{ Carphone } & 28 & 2.1590 & 0.9037 & 3.3406 & -0.3243 \\
\cline { 2 - 6 } & 32 & 2.1271 & 1.0335 & 3.7453 & -0.3882 \\
\cline { 2 - 6 } & 36 & 2.0500 & 1.0230 & 4.1852 & -0.4503 \\
\cline { 2 - 6 } & 40 & 2.0310 & 1.0434 & 5.0347 & -0.5799 \\
\hline
\end{tabular}

Figure 2 compares the actual data obtained from our experiment and the corresponding curve approximated by the proposed model in (2). The dark blue lines denote the actual data curve, whereas the red lines represent the approximated model curve. We define this curve as a "Standard Deviation-to-Rank" (SR) curve. The result shows that the proposed model is accurate enough.

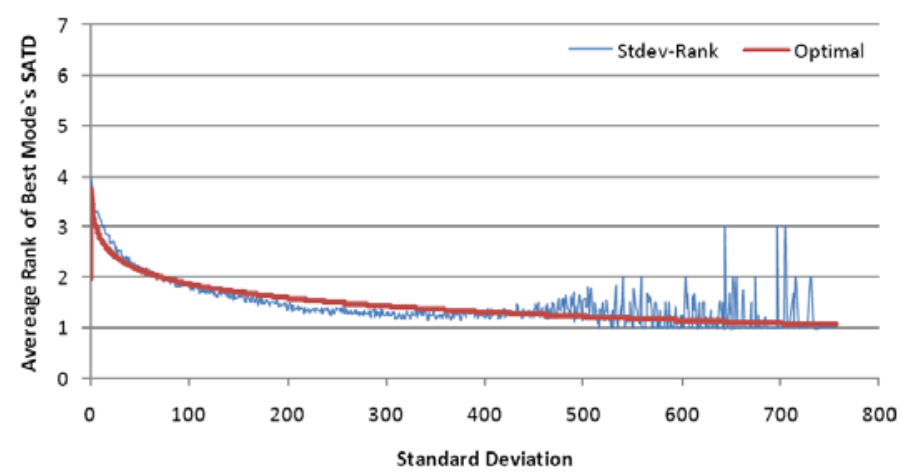

Figure 2. Comparison between the actual data and the proposed model (Foreman, QCIF, 300 I-Frames, QP = 18).

However, for each frame, the SR data of a video frame can be obtained after the frame has been encoded. This is only feasible for non-realtime coding and transcoding applications. In real-time applications, only the data of previous encoded frames are available, whereas the SR data of the current frame is unknown before performing RDO. However, we will show that the SR model of current frame can be estimated using the data of previous coded frames with fairly good accuracy according to the following observation.

3) Observation 3: Temporal similarity of SR models.

Suppose the SR data of the $j$ th frame is denoted as $S R_{j}$. And the linear regression is defined as Linest(). The model coefficients of the $j$ th frame is thus $\operatorname{Linest}\left(S R_{j}\right)$. The cumulative SR data of previous $t$ frames, $S R_{j, t}$, is defined in (4).

$$
S R_{j, t}=\sum_{i=j-1}^{j-t} S R_{i}
$$

Moreover, the curve coefficients of pervious $t$ frames is denoted as Linest $\left(S R_{j, t}\right)$. The sum of coefficient difference, $C D_{t}$, is defined as follows.

$$
C D_{t}=\sum_{i=1}^{N}\left\|\operatorname{Linest}\left(S R_{i, t}\right)-\operatorname{Linest}\left(S R_{i}\right)\right\| \text {, }
$$

where $N$ is the total number of frames. As a special case, the SR data from all frames is defined as $S R_{\text {All }}$ by using (6).

$$
S R_{\text {All }}=\sum_{i=1}^{N} S R_{i}
$$

The model parameter set of all frames is denoted as Linest $\left(S R_{A l l}\right)$. Then the sum of coefficient difference, $C D_{\text {All }}$, is defined in (7).

$$
C D_{\text {All }}=\sum_{i=1}^{N}\left\|\operatorname{Linest}\left(S R_{\text {All }}\right)-\operatorname{Linest}\left(S R_{i}\right)\right\|
$$

By using the features defined in (4), (5), (6), and (7), the temporal similarity of SR data between current frame and previous frames can be characterized. Table II shows the coefficient difference with different values of $t$. We can observe that the coefficient difference is small when $t$ is small enough, meaning that there exists temporal similarity between the SR models of current frame and previous frames. As a result, the SR curve of current frames from can be estimated form those of previous frames before encoding current frame with high reliability.

TABLE II. Coefficient Difference for Foreman QCIF

\begin{tabular}{|c|c|c|c|c|c|c|}
\hline \multirow{2}{*}{$\mathbf{Q P}$} & \multicolumn{2}{|c|}{$C D_{1}$} & \multicolumn{2}{c|}{$C D_{5}$} & \multicolumn{2}{c|}{$C D_{\text {All }}$} \\
\cline { 2 - 7 } & $\mathbf{C}$ & $\mathbf{D}$ & $\mathbf{C}$ & $\mathbf{D}$ & $\mathbf{C}$ & $\mathbf{D}$ \\
\hline 20 & 75.28 & 14.66 & 57.80 & 12.41 & 127.45 & 28.73 \\
\hline 24 & 60.53 & 11.59 & 49.13 & 10.62 & 118.96 & 27.01 \\
\hline 28 & 63.79 & 12.24 & 53.43 & 11.69 & 128.79 & 29.93 \\
\hline 32 & 82.21 & 15.28 & 67.41 & 14.12 & 140.66 & 31.88 \\
\hline 36 & 85.42 & 16.00 & 62.09 & 12.47 & 138.85 & 29.49 \\
\hline 40 & 86.98 & 17.84 & 63.44 & 13.93 & 87.27 & 18.61 \\
\hline
\end{tabular}

C. Computation Allocation

The objective of computation resource allocation is to determine the number of candidate modes for each $4 \times 4$ intra blocks. The computation budget for the $j$ th frame, Budget ${ }_{j}$, is defined as follows:

$$
\text { Budget }_{j}=\frac{\text { Buffer }}{C} \text {. }
$$

Before encoding a $4 \times 4$ intra block, we define two control parameters as below:

1) Budget: the computation budget for the ith $4 \times 4$ intra block.

2) Extra: After encoding ith $4 \times 4$ intra block, this control parameter is used to record the surplus computational budget for encoding next block. 


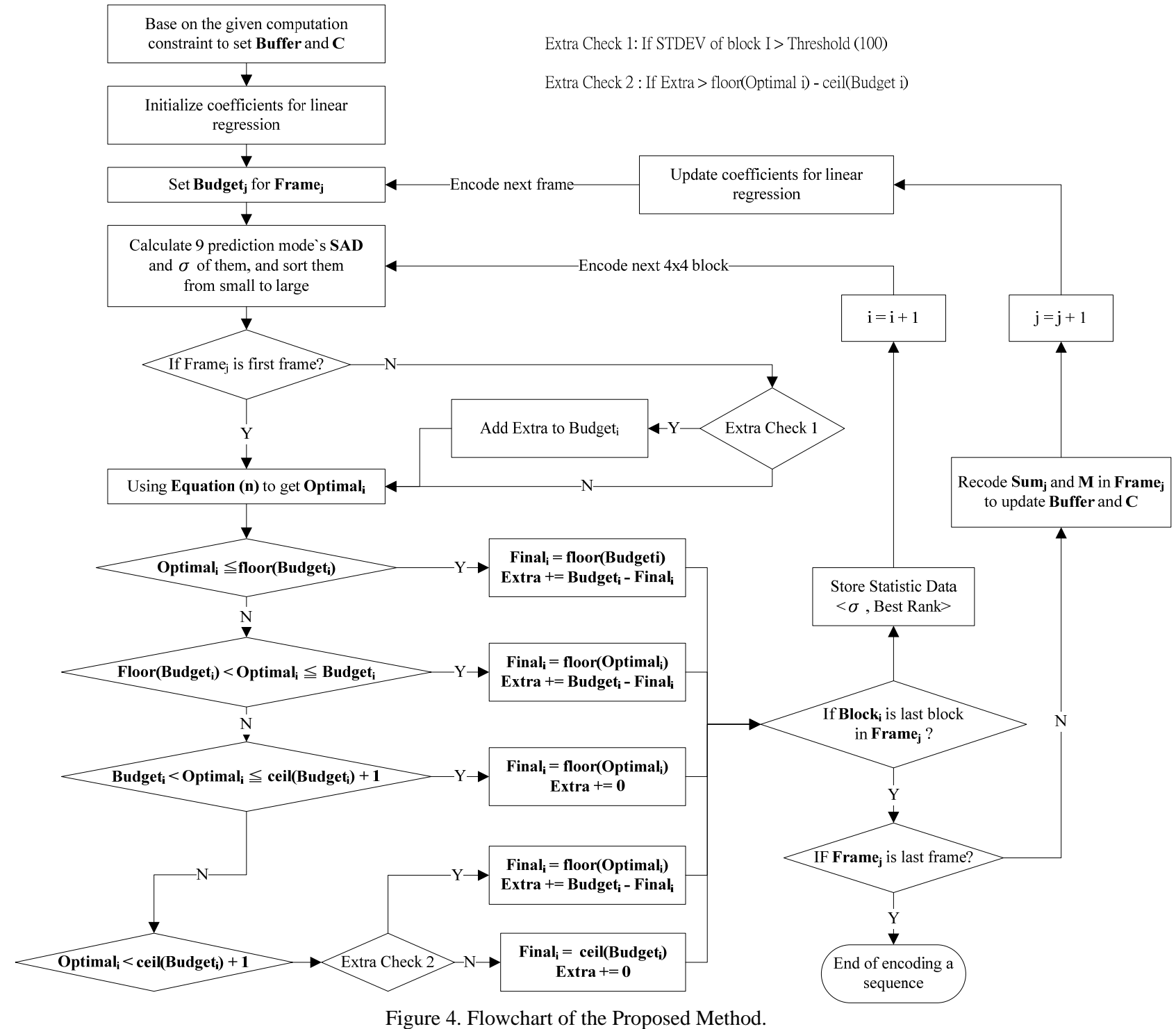

While initializing Budget ${ }_{j}$, a check of standard deviation is performed to find out additional computation according to the values of Extra. The SR model usually drops sharply at the small standard deviation region as shown in Figure 3, that is, the SR curve value is smaller than the statistical data at left region of the red vertical line. Therefore, if the standard deviation of the $i$ th block is smaller than the threshold $\left(T H_{\sigma}\right)$ and there are some extra computations, the Budget ${ }_{i}$ can be increased. The value of $\mathrm{TH}_{\sigma}$ is determined empirically.

Finally, the initialization of Budget $_{i}$ is described in (9).

$\left\{\begin{array}{c}\text { Budget }_{i}=\text { Budget }_{j}+1 \\ \text { Extra }=\text { Extra }-1\end{array}\right.$, when $\sigma<$ TH $_{\sigma}$ and Extra $>1$

$\left\{\begin{array}{l}\text { Budget }_{i}=\text { Budget }_{j} \\ \text { Extra is not change }\end{array}\right.$, otherwise

Before choosing the candidate modes for each block, as mentioned previously, the optimal number of candidate modes can be estimated based on the features of each block. Thus, in the following, Budget ${ }_{i}$ or Optimal $_{i}$ are chosen as the final number of candidate modes based on the relationship between Budget $_{i}$ or Optimal . $_{\text {. }}$

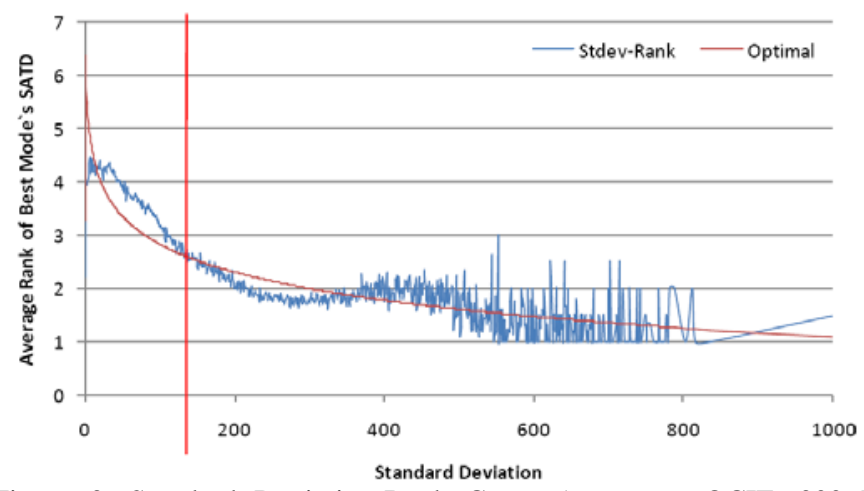

Figure 3. Standard Deviation-Rank Curve (Foreman, QCIF, 300 IFrames, QP=40) 
There are four cases of the relationship between Budget $_{i}$ and Optimal $_{i}$. The final number of candidate modes for the ith intra block is denoted as Final. In each case, the proposed method chooses Budget ${ }_{i}$ and Optimal $_{i}$ to be Final $_{i}$, and update Extra for encoding next intra block. Each case is described below:

1) Optimal $_{i} \leq\left\lfloor\right.$ Budget $\left._{i}\right\rfloor$

If Optimal $_{i}$ is much smaller than Budget ${ }_{i}$, it means that the computation budget is enough. To meet the budget as close as possible, the following updates vare performed.

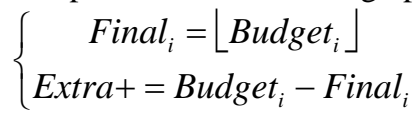

2) $\left\lfloor\right.$ Budget $\left._{i}\right\rfloor<$ Optimal $_{i} \leq$ Budget $_{i}$

If Optimal ${ }_{i}$ is slightly smaller than Budget ${ }_{i}$ Optimal $_{i}$ is prefferred to be chosen as Final $_{i}$ because the computation constraint can be met and the svaed computation can be reserved for encoding the following blocks. As a result, the computation buffer control is as follows:

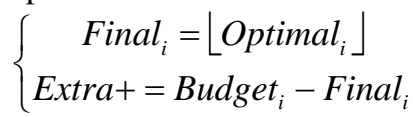

3) Budget $_{i}<$ Optimal $_{i} \leq\left\lfloor\right.$ Budget $\left._{i}\right\rfloor+1$

If Optimal $_{i}$ is slightly larger than Budget ${ }_{i}$, it is preferred to be Final $_{i}$ because the computation does not exceed the budget too much. Therefore, we update Final $_{i}$ as in (12)

4) $\left\lfloor\right.$ Budget $\left._{i}\right\rfloor+1<$ Optimal $_{i}$

$$
\text { Final }_{i}=\left\lfloor\text { Optimal }_{i}\right\rfloor
$$

If Optimal $_{i}$ is much larger than Budget ${ }_{i}$, it means that the computation budget is not enough. To meet the budget as close as possible, Final $_{i}$ is updated as follows:

$$
\text { Final }_{i}=\left\lceil\text { Budget }_{i}\right\rceil
$$

Case 4 is the worst case in the proposed method, because the available computations are too low to satisfy the optimal number of candidate modes. If there are many extra computations when case 4 is chosen, then it can change to case 3 to fit the optimal value of candidate modes.

$$
\left\{\begin{array}{c}
\text { Final }_{i}=\left\lfloor\text { Optimal }_{i}\right\rfloor, \text { when Extra }>\left(\left\lfloor\text { Optimal }_{i}\right\rfloor-\left\lceil\text { Budget }_{i}\right\rceil\right) \\
\text { Final }_{i}=\left\lceil\text { Budget }_{i}\right\rceil, \text { otherwise }
\end{array}\right.
$$

\section{Computational Buffer Update}

After encoding all $4 \times 4$ intra blocks in the $j$ th frame, we define $\mathrm{Sum}_{j}$ which is used to record the total number of candidate modes for RDO computation for the $j$ th frame.

$$
\text { Sum }_{j}=\sum_{i=0}^{M-1} \text { Final }_{i},
$$

where $M$ is the total number of $4 \times 4$ blocks in the $j$ th frame. Subsequently we update the two control parameters Buffer and $C$ in the computation buffer as in (16) for encoding the next frame.

$$
\left\{\begin{array}{c}
\text { Buffer }=\text { Buffer }- \text { Sum }_{j} \\
C=C-M
\end{array}\right.
$$

The flowchart of the proposed computation control algorithm is depicted in Figure 4.

\section{EXPERIMENTAL RESULTS}

The proposed computation control algorithm is implemented into JVT JM 12.2 reference software, and compared with the original H.264 encoder with full search. In our experiments, the environment settings are as follow:

- Intra Period is set to 1. (All frame using intra coding only)

- Main profile is adopted with RDO and CABAC enabled.

- Each 300-frame sequence is encoded with four quantization parameters: 20, 24, 28, 32, 36, and 40.

- $\quad T H_{\sigma}=1$ for each test sequences.

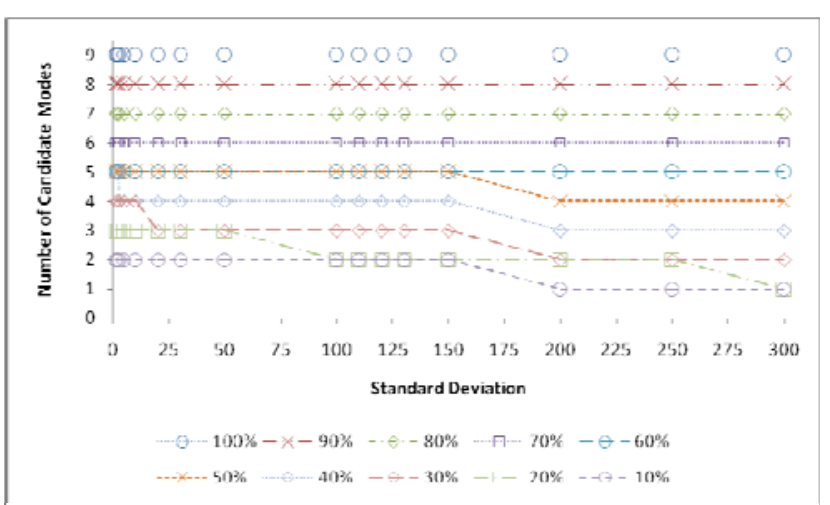

(a)

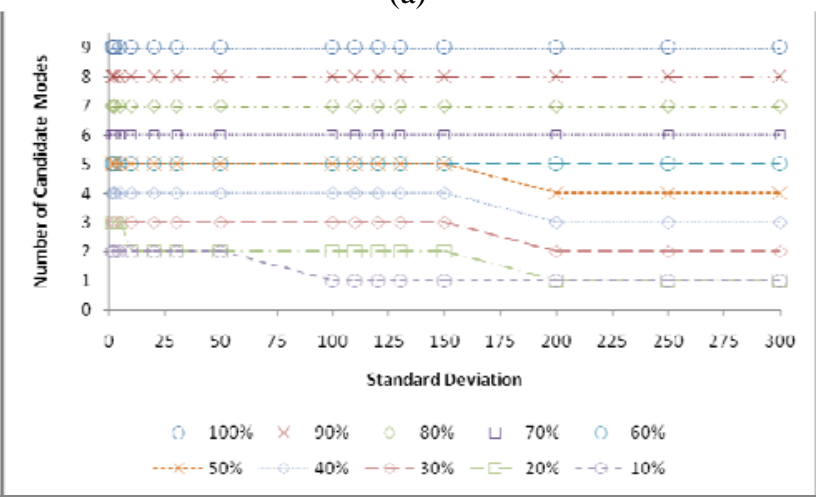

(b)

Figure 5. Average number of candidate modes using the proposed algorithm for QCIF sequences: (a) Foreman, $\mathrm{QP}=40$, (c,d) $=(5.45,-0.61)$; (b) Coastguard, $\mathrm{QP}=20,(c, d)=(3.73,-0.42))$

Figure 5 shows the average number of candidate modes by using our proposed method with various constraints. Under each computation constrain, the proposed algorithm always makes a decision that fits the trend of SR curve and assigns the computation resources properly. Table III lists the average time saving with various computation constraints. The time of intra $4 \times 4$ mode decision consumes about $60 \%$ of the total encoding time according to the experiments, and our algorithm almost fit this condition with the optimal coding efficiency.

Figure 6 to Figure 8 show the RD-performance results. We compare the proposed method with three different approaches. The blue square lines indicate the RD-performance of the original H.264 encoder. The light orange triangles represent the method that uses the fixed number of candidate modes in the encoding procedure. The fixed numbers of prediction modes are determined by the constraints. For example, if the computation 
constraint is $50 \%$, the fixed number of prediction modes is 4.5 on average. The red diamond lines show the modified H.264 encoder that use only the DC mode and disables all other $4 \times 4$ intra mode for intra $4 \times 4$ blocks. According to the PSNR comparison, we can see that ever though only $20 \%$ computation constraint is given, compared with the original H.264 encoder, the PSNR loss of the proposed method is only smaller than 0.24 $\mathrm{dB}$ in the worst case. This means that our computational control algorithm can maintain the acceptable video quality when the computational resource is limited.

Table III. Average Time Saving with various computation constrains

\begin{tabular}{|c|c|c|c|c|}
\hline Sequences & $80 \%$ & $60 \%$ & $40 \%$ & $20 \%$ \\
\hline Foreman & $12.55 \%$ & $25.35 \%$ & $34.94 \%$ & $48.65 \%$ \\
\hline Akiyo & $11.92 \%$ & $25.21 \%$ & $34.02 \%$ & $47.85 \%$ \\
\hline Carphone & $12.16 \%$ & $25.61 \%$ & $34.53 \%$ & $48.58 \%$ \\
\hline Coastguard & $13.12 \%$ & $26.85 \%$ & $35.62 \%$ & $50.09 \%$ \\
\hline
\end{tabular}

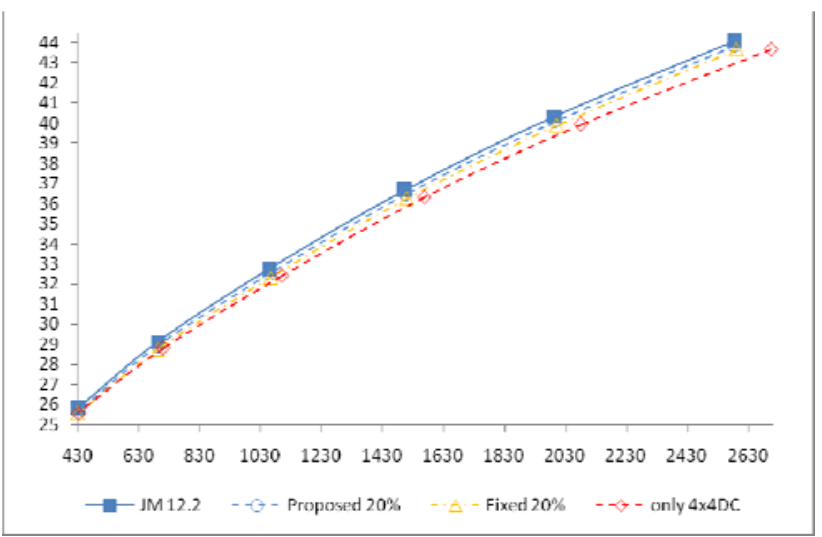

Figure 6. The RD-performance comparison between the original H.264 encoder with proposed computation control algorithm. (Stefan, QCIF, 300 I-Frames)

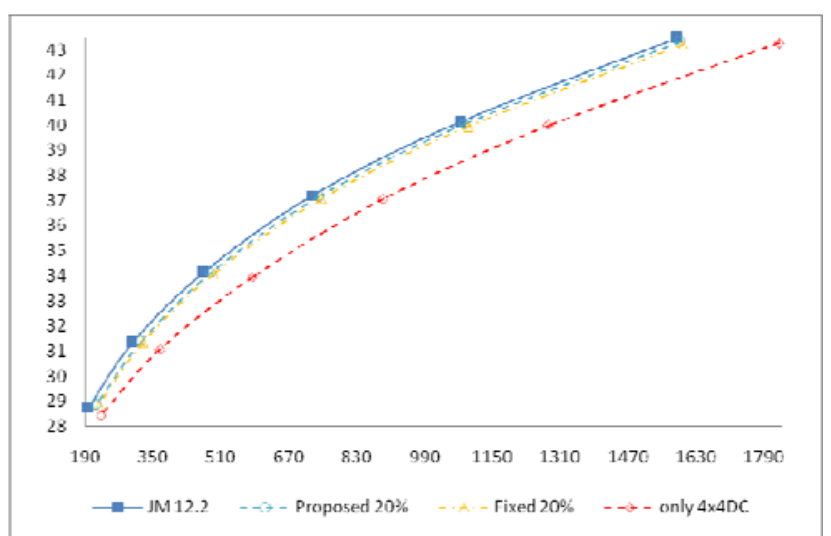

Figure 7. The RD-performance comparison between the original H.264 encoder with proposed computation control algorithm. (Foreman, QCIF, 300 I-Frames)

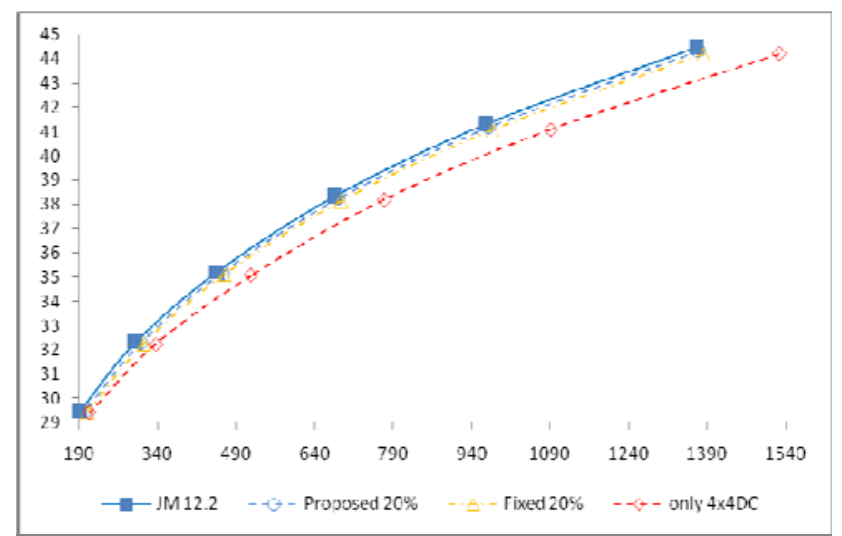

Figure 8. The RD-performance comparison between the original H.264 encoder with proposed computation control algorithm. (Carphone, QCIF, 300 I-Frames)

\section{CONCLUSIONS}

We proposed a computation-aware intra mode decision for H.264 coding and transcoding applications. The proposed algorithm optimizes the visual quality by adaptively adjusting the number of prediction modes in mode decision under a given computation constraint. We introduced a new concept of computation buffer and formulate the computation control of mode decision as a rate-distortion optimization problem of computation buffer control. Experimental results show that our proposed algorithm can effectively control the computational complexity while maintaining good RD-performance and satisfying the given computation constraint.

\section{REFERENCE}

[1] T. Wiegand, G. J. Sullivan, G. Bjontegaard, and A. Luthra, "Overview of the H.264/AVC video coding standard," IEEE Trans. Circuits Sys. Video Technol., vol. 13, no. 7, pp. 560-576, July 2003.

[2] C. S. Kannangara, I. E. G. Richardson, M. Bystrom, J.R. Solera, Y. Zhao, A. MacLennan, and R. Cooney, "Lowcomplexity Skip Prediction for H.264 Through Lagrangian Cost Estimation,” IEEE Trans. Circuits Sys. Video Technol., vol. 16, no. 2, pp. 202-208, Feb. 2006.

[3] C. S. Kannangara and I. E. G. Richardson, "Computational control of an H.264 encoder through Lagrangian cost function estimation," in Int. Workshop Very Low Bit-rate Video-coding, Sept. 2005.

[4] Y. Wang and S.-F. Chang, "Complexity adaptive H.264 encoding for light weight streams," Proc. IEEE Int. Conf. Acoustics, Speech Signal Processing, May 2006. 